\title{
Performance Evaluation of 60-GHz-WPAN System Distributed Over Multi-Mode Fiber
}

\author{
Moussa El Yahyaoui, Ali El Moussati and Kamel Haddadi
}

\begin{abstract}
The manuscript deals with the assessment of Radio over Fiber (RoF) system including pure electrical baseband, pure radio frequency band centered around $60 \mathrm{GHz}$, and hybrid radiooptical system at the same RF band using a global simulation. In this work we focus on RoF solution to improve the low coverage of the $60 \mathrm{GHz}$ channel caused by high free-space attenuation. A realistic co-simulation of the Wireless Personal Area Network (WPAN) IEEE802.15.3c-RoF was performed in a residential environment for Line-Of-Sight (LOS) and Non-LineOf-Sight (NLOS). In this work, we demonstrated a $60 \mathrm{GHz}$ radio on Multi-Mode Fiber (MMF) using Optical Carrier Suppression (OCS) modulation. The BER (Bit Error Ratio) performance of this system is measured by varying the following parameters: optical launched power, fiber length, modulation format, Channel coding and Signal to Noise Ratio. We show that the RoF at 60 GHz can reach a minimum of $300 \mathrm{~m}$ of MMF without optical amplifiers followed by a $5 \mathrm{~m}$ wireless transmission with BER less than $10^{-3}$ in the LOS and NLOS environments.
\end{abstract}

Keywords-60 GHz, MMF, OCS, RoF, Wireless channel model TSV.

\section{INTRODUCTION}

M ODERN telecommunication applications require data rate in the order of multi $\mathrm{Gb} / \mathrm{s}$ such as uncompressed Video Streaming, Wireless Display, Gaming and High Capacity disc drive synchronization. In order to tackle this challenge, much particular attention has been recently accorded to 60 $\mathrm{GHz}$ Band Millimeter Wave Technology (MWT), which can offer throughput up to $7 \mathrm{~Gb} / \mathrm{s}$. In particular, standards such as IEEE802.15.3c [1], ECMA-387 [2], Wireless-HD [3] and IEEE802.11ad [4] systems have been introduced to work at $60 \mathrm{GHz}$. However, the operating frequency $60 \mathrm{GHz}$ presents high free space attenuation and cannot therefore penetrate the walls, which limits the coverage to few meters (i.e. single room). This implies the deployment of multiple radio access points in a single house for a complete coverage. In order to extend the coverage of $60 \mathrm{GHz}$, the Radio over Fiber (RoF) solution has been introduced [5].

Combining the high bandwidth of optical fiber with the flexibility of wireless access, RoF systems become more attractive in $60 \mathrm{GHz}$ wireless communications. In addition, RoF can reduce the deployment and maintenance cost of wireless access networks, as well as the energy consumption. The transport of the radio signal from the central station to the Remote Unit (RU) can be performed at baseband, at

M. El Yahyaoui and A. El Moussati are with department Electronics, Informatics and Telecommunications, Ecole Nationale des Sciences Appliquees, ENSAO, Morocco (e-mail: \{m.elyahyaoui, a.elmoussati\}@ump.ac.ma).

$\mathrm{K}$. Haddadi is with IEMN-Institute of Electronics, Microelectronics and Nanotechnology, University Lille, France. intermediate frequency (IF) or directly at radio frequency (RF) over optical fiber [6]. Different optical fiber types such as SMF, MMF, and plastic optical fiber are used in RoF system [7],[8],[9]. RoF over MMF allows the use of low cost and largely available optoelectronic devices working at $850 \mathrm{~nm}$. Moreover, MMF is installed in the most new building [10]. However, the use of MMF is confined to very short links due to modal bandwidth limit imposed by bandwidth-distance product. To overcame this limitation, the authors in [11] employ the Optical Frequency Multiplication (OFM) technique to transmit RF up to $40 \mathrm{GHz}$ over MMF, and the authors in [12] have turbo code to transmit $50 \mathrm{GHz}$ over MMF over 300 $\mathrm{m}$ of fiber. In our work, we propose the OCS modulation with Low Density Parity Check (LDPC) codes to transmit $60 \mathrm{GHz}$ over MMF.

In this work, we propose a hybrid optical-wireless communication system at $60 \mathrm{GHz}$. The wireless subsystem considered is the physical layer of the standard IEEE802.15.3c, which uses two robust technologies; Orthogonal Frequency Division Multiplexing (OFDM) modulation and forward error correction LDPC codes [13]. In addition, this system uses the Frequency Domain Equalizer (FDE) to overcome the nonlinear and modal dispersion of MMF [14]. The optical subsystem is based on OCS modulation as a solution to increase the MMF length. The $60 \mathrm{GHz}$ channel model is performed by TSV (Triple S and Valenzuela) model, built by Task Group 802.15.3c (TG3c), which considers the multi-path phenomenon caused by reflection, scattering and diffraction of radio waves during the transmission [15], [16]. The proposed system is evaluated using both MATLAB/Simulink and OptiSystem co-simulations.

The rest of this paper is organized as follows: Section 2 gives an overview of RoF system at $60 \mathrm{GHz}$; Section 3 presents the setup simulation and in Section 4, we evaluate the system performance in terms of BER with fiber length, Energy bit to Noise ratio (EbNo), Distance of the antennas, and various modulation schemes.

\section{RAdio OVER Fiber System AT $60 \mathrm{GHz}$}

In home area networks, the signal generation and processing are centralized in the Home Communication Controller (HCC). The radio signals are transported in their original format, which simplify the remote antenna architecture and provide transparency to radio layer protocols. The radio access control, signal generation and processing are carried out at a centralized HCC and the signals are delivered to the simplified remote antennas that contain only RF modules via optical distribution 


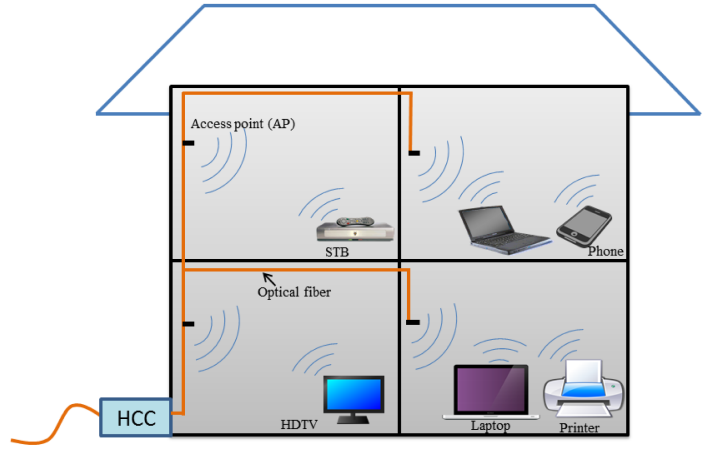

Fig. 1. RoF home area network architecture

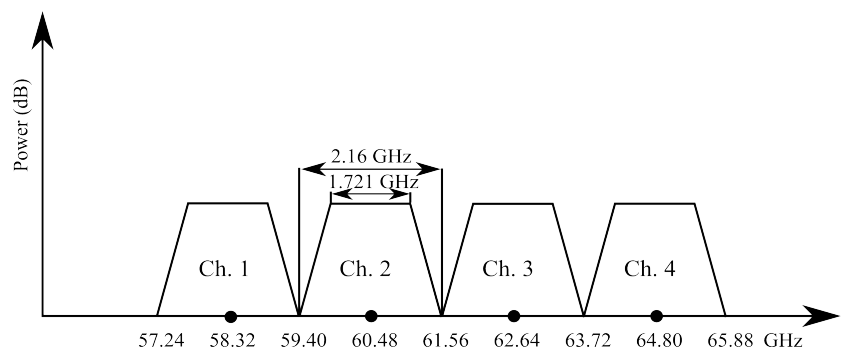

Fig. 2. Channelization of the $57-66 \mathrm{GHz}$ band

network [17]. A simple example of a RoF system in home area network is shown in Fig. 1.

\section{A. IEEE802.15.3c HSI Physical Layer Transceiver}

In standard IEEE 802.15.c, the MWT physical layer is defined within the frequency band of 57-66 GHz, which consists of four channels of $2.16 \mathrm{GHz}$ as shown in Fig. 2 . Three different physical layer modes are defined as Single Carrier (SC), High Speed Interface (HSI), and Audio-Visual (AV) modes. In our work, we consider the HSI mode, which offers high data rate (e.g. up to $6 \mathrm{~Gb} / \mathrm{s}$ ) and that is designed for NLOS operation using OFDM and LDPC channel coding. It provides NLOS high speed, low-latency communication and bidirectional communication.

In order to evaluate the performance of $60 \mathrm{GHz}$ wireless transceiver, we have implemented the $60 \mathrm{GHz}$ physical layer in MATLAB as depicted in Fig. 3. The data bit stream is coded by means of two parallel LDPC encoders. Interleavers are added in order to protect the transmission against burst errors. Then the data are sent to the symbol mapper, which maps the input bits into QPSK, 16QAM and 64QAM symbols depending on the modulation scheme. The output data of the constellation mapper are then parallelized, and DC, null, pilot tones and training sequence are added up. The pilot tones are used for frame detection and carrier frequency offset estimation and the training sequence used for channel estimation. The nulls subcarriers are unused in order to allow a low-pass filtering. The Inverse Fast Fourier Transform (IFFT) operation (size 512) is then applied to the resulting stream in order to determine the OFDM symbols. In order to improve the immunity to inter-symbol interferences, a cyclic prefix consisting of the last 64 samples of the symbol is inserted at

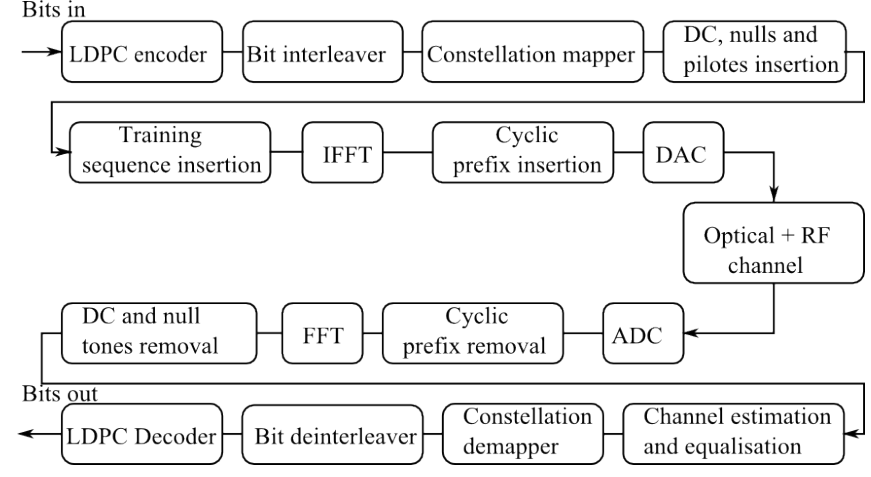

Fig. 3. Block diagram of the 60-GHz IEEE802.15.3c HSI physical layer implemented in MATLAB/Simulink

the beginning of the OFDM symbol itself [18]. The Digitalto-Analog Converter (DAC) and Analog-to-Digital Converter (ADC) are modeled in Simulink using a raised cosine filter with a roll-off factor of 0.2 . At the receiver, after downconversion and filtering processes, the cyclic prefix is removed from the OFDM symbol, and the Fast Fourier Transform (FFT) operation is carried out on the received stream. A block of channel estimation and gain correction is used to overcome the sensitivity of the de-mapper to the amplitude of the input symbols. The channel response is estimated by extracting the received training sequence values and dividing them by the expected training sequence values,

$$
H(k)=S_{R x}(k) / S_{T x}
$$

where, $k$ is the training sequence index, $S_{R x}$ are the amplitude values of received training sequence and $S_{T x}$ are the amplitude values of the expected training sequence. Then, the FDE is applied using Zero Forcing (ZF) algorithm, in which the gain is defined as the inverse of the channel frequency response $H$. The restored signal is obtained by the product of the received signal and equalizer gain.

\section{B. IEEE802.15.3c Wireless Channel Model}

The TG3c developed a MWT channel model to evaluate the performance of different physical layer proposals [16]. This model is named TSV, and is constructed by integrating the two-path model with the SV model [19]; it includes a direct path LOS and Angle of Arrival (AoA) in addition to the SV-model which has been used for WLAN. This model is based on a lot of measured data and statistical analysis [15],[16], which suitably described the large-scale fading and small-scale fading characteristics for $60 \mathrm{GHz}$ wireless channel. A schematic typical impulse response generated by the TSV model is shown in Fig. 4. The first response indicates the two-path model response (LOS path) and the other responses indicate SV model response due to NLOS paths. where, $\Lambda$ is cluster arrival rate, $\lambda$ is ray arrival rate, $\Gamma$ is cluster decay factor and $\gamma$ is ray decay factor.

The Channel Impulse Responses (CIR) of TSV model is given 


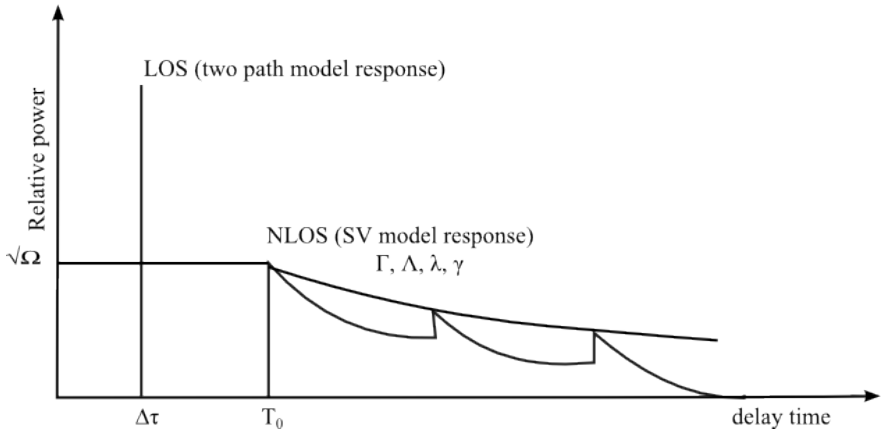

Fig. 4. Impulse response of TSV channel model

by

$h(t)=\beta \delta(t)+\sum_{l=0}^{L-1} \sum_{m=0}^{M_{l}-1} \alpha_{l, m} \delta\left(t-T_{l}-\tau_{l, m}\right) \delta\left(\phi-\Psi_{l}-\psi_{l, m}\right)$

with

$\delta$ : Delta function.

$\beta$ : Amplitude of the component Line Of Sight (LOS), which contains information about the antenna height of the transmitter and receiver, the distance between the antennas. $\alpha_{l, m}$ : Complex amplitude of the $m^{t h}$ ray of the $l^{t h}$ cluster. $T_{l}$ : Delay time of the $l^{\text {th }}$ cluster.

$\tau_{l, m}$ : Delay time of the $m^{\text {th }}$ ray in $l^{\text {th }}$ cluster.

$\Psi_{l}$ : Angle of arrival of the $l^{\text {th }}$ cluster.

$\psi_{l, m}$ : Angle of arrival of $m^{t h}$ ray in the $l^{t h}$ cluster.

This model was approved as TG3c's general channel model [16] and its program codes (MATLAB program) were developed and uploaded by the authors to the IEEE server [20] for public use. TG3c developed various environment channels model in LOS and NLOS conditions. This paper employs two kinds of indoor environment channel models recommended by TG3c: indoor LOS channel (CM1) and indoor NLOS channel (CM2), respectively. The generation of CIR is based on MATLAB program previously mentioned.

\section{Optical Fiber Link}

The principle of $60 \mathrm{GHz}$ RoF based system on OCS modulation is presented in Fig. 5 . The In-phase (I) and Quadrature (Q) components are transposed to Intermediate Frequency (IF) set to $5 \mathrm{GHz}$ as shown in Fig. 6 to support multi-users using Subcarrier Multiplexing (SCM) technique [6]. This IF signal is modulated onto an optical carrier frequency using first MachZehnder Modulator (MZM1). The optical carrier frequency generated by a Continuous-Wavelength (CW) optical source of $850 \mathrm{~nm}$ wavelength. The OCS modulation is performed by polarizing the second Mach-Zehnder Modulator (MZM2) to its minimum intensity and driving the two arms with 27.74 $\mathrm{GHz}$ signal phase-shifted by $\pi$ [21]. The input optical field to MZM2 can be described as

$$
E_{\text {in }}(t)=E_{0} s(t) \exp \left[j 2 \pi f_{c} t+j \varphi(t)\right]
$$

where, $E_{0}$ is the amplitude of the optical carrier and $s(t)$ is the IF signal. The MZM2 is driven by an RF signal, the optical

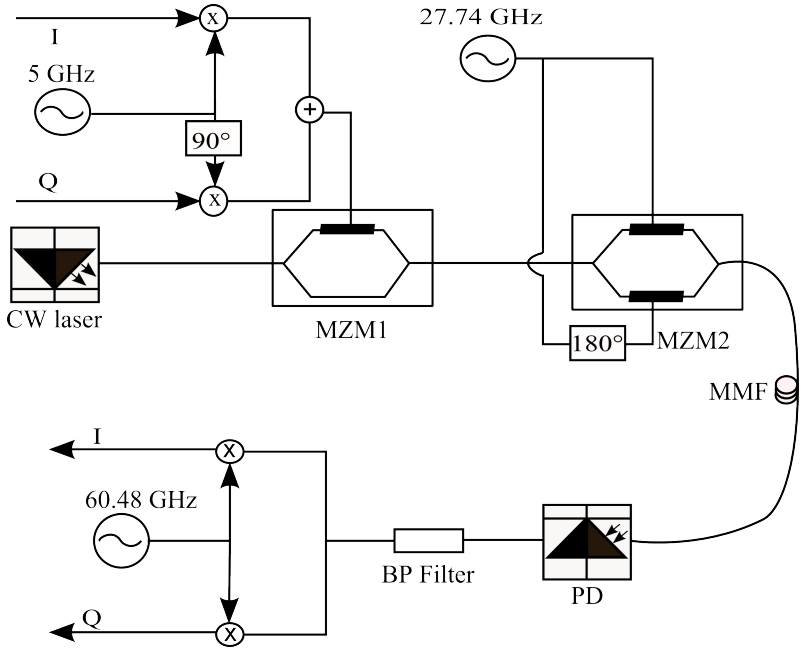

Fig. 5. $60 \mathrm{GHz}$ RoF architecture with OCS modulation

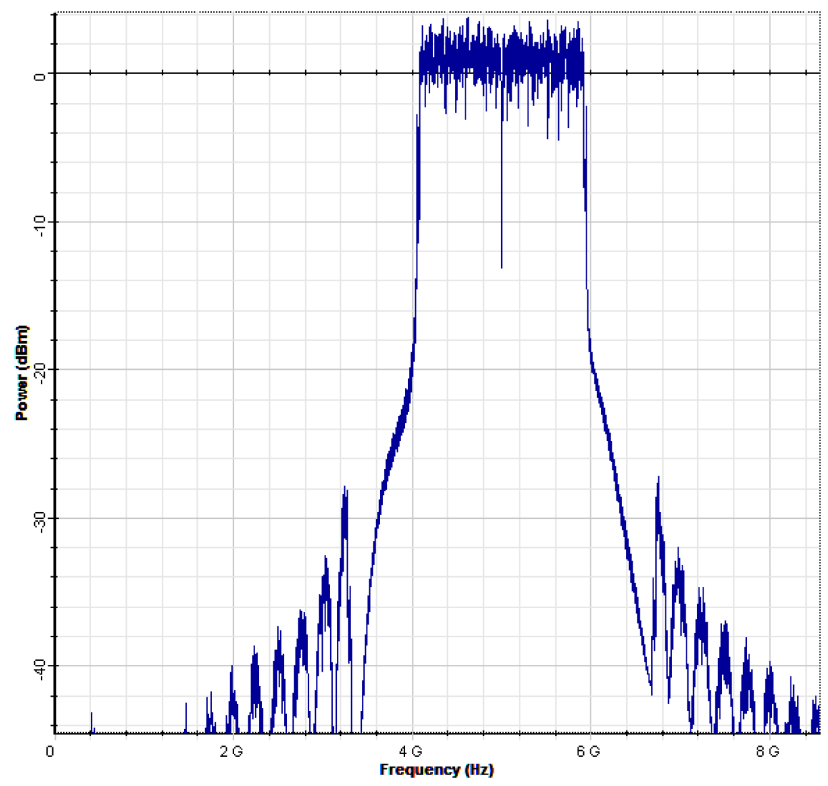

Fig. 6. OFDM signal spectrum at $5 \mathrm{GHz}$

output can be written as

$$
\begin{gathered}
E_{\text {out }}(t)=E_{0} s(t) \sum_{n=1}^{\infty}(-1)^{n} J_{2 n-1}(m)\left\{\operatorname { e x p } \left[j 2 \pi f_{c} t+\right.\right. \\
\left.j(4 n-2) \pi f_{L O}+j \varphi(t)\right]+\exp \left[j 2 \pi f_{c} t-\right. \\
\left.\left.j(4 n-2) \pi f_{L O}+j \varphi(t)\right]\right\}
\end{gathered}
$$

$j_{n}$ denotes the $n^{t h}$ order Bessel function, and $m$ is the phase modulation index $m=\pi V_{m} / V_{\pi}$. To keep the side bands signals with suppressed carrier the modulator MZM2 should be polarized to its minimum intensity $V_{\pi}\left(V_{m}=V_{\pi}\right)$. Hence, by considering only the first order sideband the Equation 4 


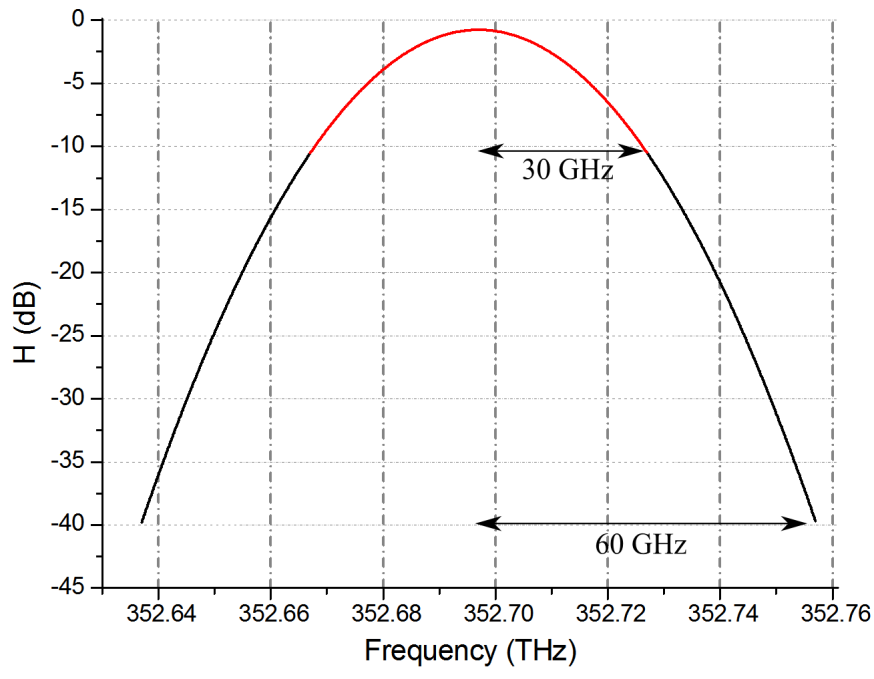

Fig. 7. The transmission function of $300 \mathrm{~m}$ of OM4 MMF

can be simplified to

$$
\begin{aligned}
E_{\text {out }}(t)= & -E_{0} s(t) J_{1}(m)\left\{\exp \left[j 2 \pi f_{c} t+j(2) \pi f_{L O}+j \varphi(t)\right]\right. \\
& \left.+\exp \left[j 2 \pi f_{c} t-j(2) \pi f_{L O}+j \varphi(t)\right]\right\}
\end{aligned}
$$

The optical signal is transported by OM4 MMF, with modal bandwidth of $5000 \mathrm{MHz} . \mathrm{Km}$, to the access point [21]. The modal bandwidth measurement can be obtained by calculation of the transmission function (frequency response) $H(f)=$ $R(f) / T(f)$, where $R(f)$ is the received signal and $T(F)$ is the transmitted signal. The function transmission obtained for $300 \mathrm{~m} \mathrm{MMF}$ with120 GHz frequency range centered on 352.697 THZ $(850 \mathrm{~nm})$ is shown in Fig. 7. The side bands at $30 \mathrm{GHz}$ are attenuated by $11 \mathrm{~dB}$, while the side bands of 60 $\mathrm{GHz}$ are attenuated by $40 \mathrm{~dB}$. By using OCS modulation we can obtain an RF signal $60 \mathrm{GHz}$ with $11 \mathrm{~dB}$ instead of $40 \mathrm{~dB}$ with double sides lateral with carrier. At the receiver side, the Photo-detector (PD) converts the optical signal to electrical signal. This signal is then amplified, filtered and demodulated to recover I and Q HSI OFDM signal.

\section{Setup Simulation}

The simulation setup of the WPAN HSI-OFDM RoF system at $60 \mathrm{GHz}$ is shown in Fig. 8. The co-simulation, SimulinkOptiSystem, allows the use of optical components of OptiSystem in Simulink Software [13]. Simulink program runs an OptiSystem subroutine that contains the optical model. Each time OptiSystem subroutine is called, the model generates the data that corresponds to the input signal after the optical modulation and transmission over MMF. A signal HSI-OFDM based on standard IEEE802.15.3c is generated under Simulink. The FFT is performed on blocks of 512 points, 336 are data sub-carrier modulated in QPSK, 16QAM or 64QAM. Sample frequency of baseband system is $2.64 \mathrm{GS} / \mathrm{s}$, which is corresponding to $1.83 \mathrm{GHz}$ bandwidth. The optical IF HSI-OFDM signal generated from MZM1 is used as the optical input to MZM2 which is configured to enable optical

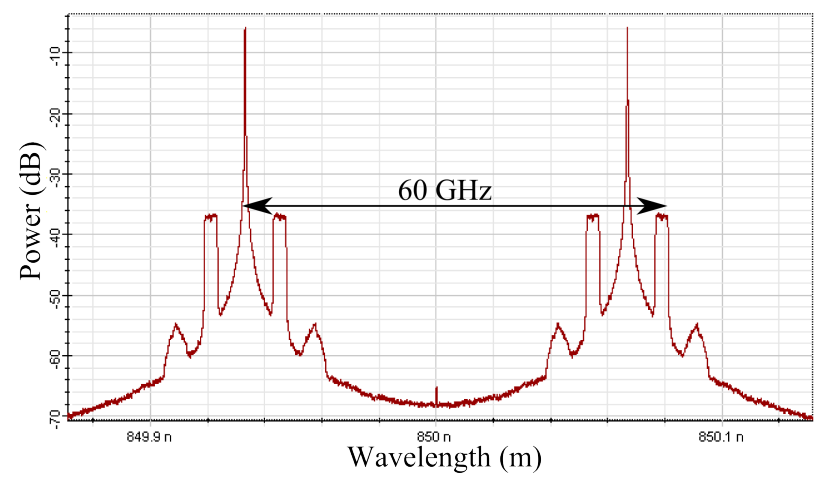

Fig. 9. Optical spectrum at output of MZM2

TABLE I

PARAMETERS OF Simulated SyStem

\begin{tabular}{cc}
\hline Parameter name & Value \\
\hline FFT length & 512 \\
Data subcarrier & 336 \\
Pilots & 16 \\
DC & 3 \\
Reserved & 16 \\
Cyclic prefix & 46 \\
Frequency sampling & $2.64 \mathrm{GHz}$ \\
Centered carrier frequency & $60.48 \mathrm{GHz}$ \\
Bandwidth & $1.83 \mathrm{GHz}$ \\
Optical fiber & $820 \mathrm{~nm}, \mathrm{MMF} \mathrm{OM} 4$ \\
Wireless distance & $5 \mathrm{~m}$ \\
\hline
\end{tabular}

carrier suppression. MZM2 is driven by a local oscillator at a frequency of $27.74 \mathrm{GHz}$. The output optical signal from MZM2 is shown in Fig. 9. We can see that there is a $60.48 \mathrm{GHz}$ separation between the optical carrier and the HSI-OFDM signal. The modulated Light-wave from MZM 2 is sent through OM4-MMF. The optical signal is then directly detected by a photo-diode with $1 \mathrm{~A} / \mathrm{W}$ responsivity and the $60 \mathrm{GHz}$ is generated by heterodyne detection. After photo-detection, the HSI-OFDM signal is amplified and downconverted by mixing with a local oscillator with frequency set to $60.48 \mathrm{GHz}$. The down-converted HSI-OFDM signal is sent through baseband TG3c channel, and then is passed to the HSI-OFDM receiver for demodulation and, BER and EVM calculation. Due to simulation loop, the number of transmitted bits is not limited; however the simulation can be stopped manually when the BER becomes stable. The parameters of the system are summarized in Table I.

\section{Results And Discussions}

Considering the complete downlink RoF system, we have simulated the wireless optic system using co-simulation between OptiSystem software from Optiwave for the optic link and Matlab/Simulink for the electrical link. To evaluate the performance of such a system, we found that several parameters such as the injected optical power, the distance of the fiber, the type of the radio propagation environment and the modulation and coding schemes can influence the results. Simulation results are carried out in order to study 


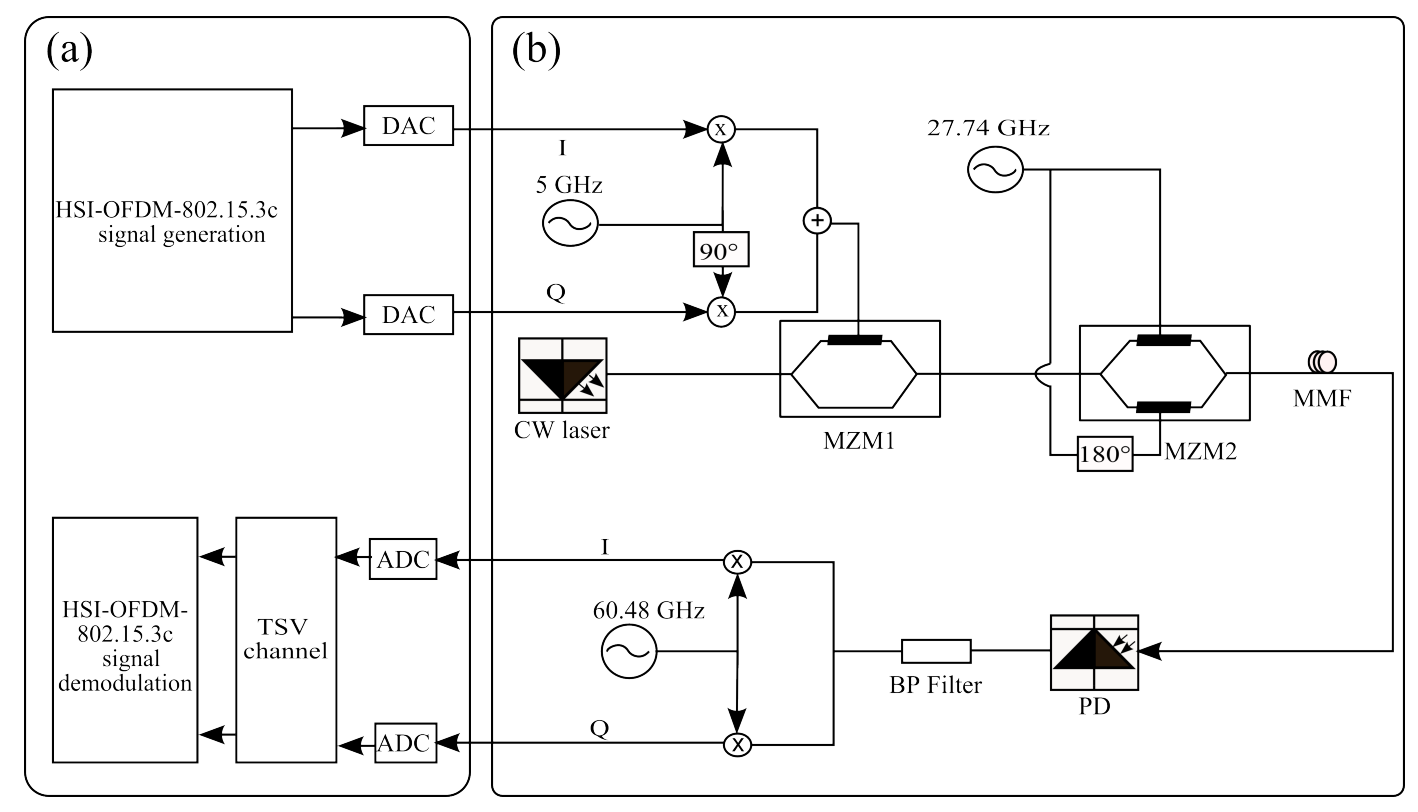

Fig. 8. Simulation schematic of complete system in (a) SIMULINK and (b) OptiSystem

the RoF system in different scenarios. We started by using the optical channel only, then we combined the two channels together. These steps can be classified into four different cases. In the first case, without RF channel, the output is observed by varying the launched optical power to select the optimum value of the injected optical power. In the second case, with $\mathrm{RF}$ channel, the output is observed by varying EbNo of RF channel with different distance of fiber, in order to choose the optimum value of the fiber distance for the remaining cases. In the third case, optical fiber length is constant, the output is observed by varying EbNo of RF channel in indoor LOS and NLOS environments. Finally, optical fiber length is constant, the output is observed by varying EbNo of RF channel and modulation and coding schemes. In all cases, Tx-Rx distance (e.g. the distance between the transmitter and the receiver) of the RF channel is invariable.

\section{A. Effect of Launched Optical Power}

In this case, we only consider the optical link to determine the optimum value of the injected optical power. We considered an MMF fiber with a length of $300 \mathrm{~m}$ using QPSK modulation. We calculated the Error Vector Magnitude (EVM) at the transmitter system in function of launched optical in Fig 10. The IEEE 802.15.3C defines EVM limits for application classes [1]. The required EVM at the transmitter is about $14 \mathrm{~dB}(20 \%)$ for QPSK modulation. The WPAN standard assumes channel correction for each modulation format which reduces greatly the probability of mistaken bits and protects propagated signal against radio channel impairment. In Fig. 10 , an injected power of the order of $11 \mathrm{dBm}$ is observed for an EVM equal to $20 \%$. For this reason we have chosen an injected power of the order $12 \mathrm{dBm}$ in the simulations of our work.

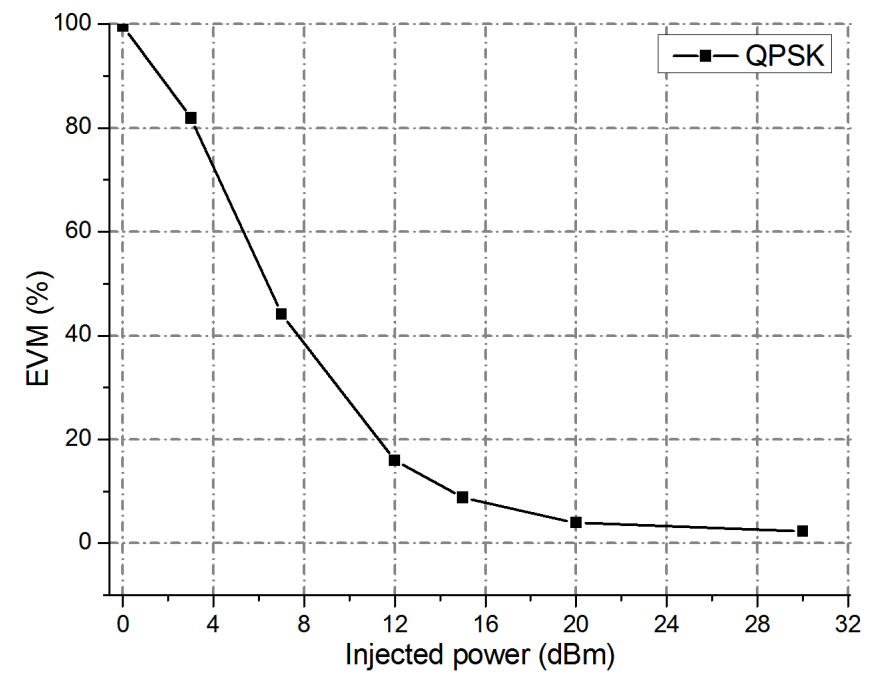

Fig. 10. EVM performances of system versus launched optical power

\section{B. Effect of Fiber Distance}

The length of the fiber and the RF propagation environment greatly influence the performance of communication systems. In order to minimize the influence of optical fiber on the performance of our system, we seek a range of the optical fiber length where the BER become independent of fiber length ( i.e. transparency of the optical fiber). Firstly, we simulated our system without introducing the RF channel. Fig. 11 presents the evolution of the BER as a function of the length of the fiber. We note that for distances less than $360 \mathrm{~m}$, the BER converges to very low values. Secondly, we evaluated the influence of fiber length on system performance with an RF channel (Distance Tx-Rx $=5 \mathrm{~m}$ ) using the NLOS mode to quantify the performance in worst case conditions. In Fig. 12, we confirm, that for lengths between $200 \mathrm{~m}$ and $300 \mathrm{~m}$, the performance remains almost invariant. For this reason, through 


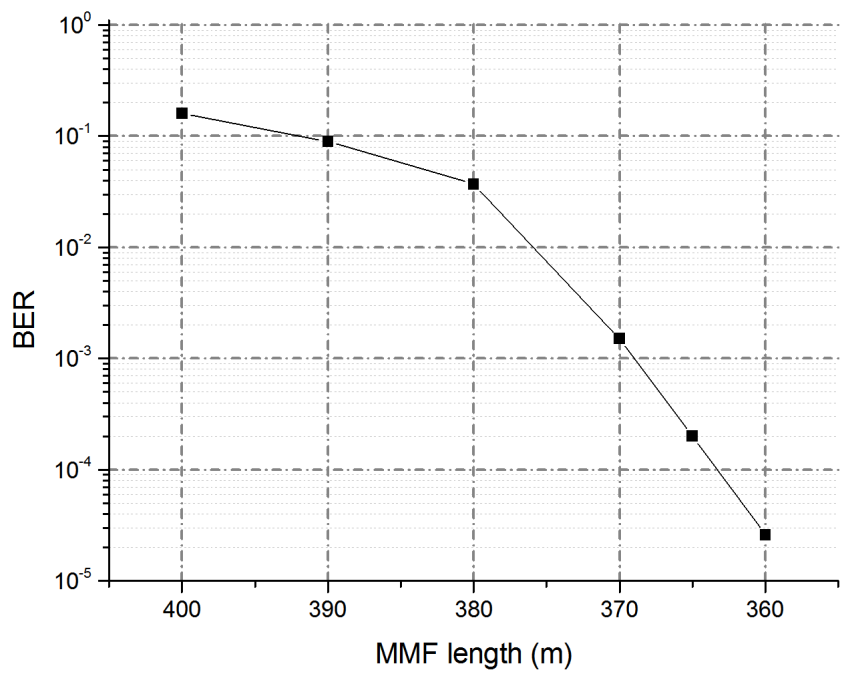

Fig. 11. BER performance versus MMF length without RF channel

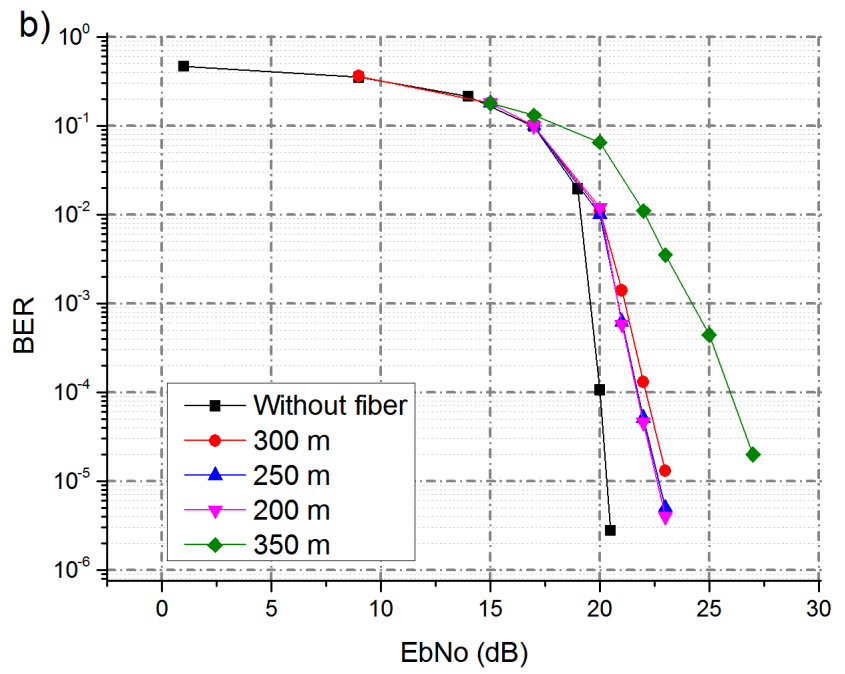

Fig. 12. BER performance versus EbNo with RF channel and different lengths of fiber

this work, we set the length of the optical fiber to $300 \mathrm{~m}$ in order to achieve acceptable performance in LOS and NLOS.

\section{Effect of Radio Transmission Medium}

Given the above model, the specific parameters for the CM1 and CM2 channel models are given in Table II. Note that the Channel models CM1 (LOS) and CM2 (NLOS) consist of 5 Transmit/receive antenna configurations in residential environment. We chose the configurations CM1.1 and CM2.1 with Half-Power Beam With (HPBW) of approximately 360 at the transmitter and 15 at the receiver. The gain of the antennas is of the order of $7 \mathrm{dBi}$. The NLOS Model CM2 can be obtained by removing the LOS path component from CM1 model.

Fig. 13 shows the variation of the BER as a function of EbNo for a LOS and NLOS scenario in a residential environment (the communication distance is $5 \mathrm{~m}$ ). We observe on the figure that $\mathrm{CM} 2$ channel is much more severe than
TABLE II

PARAMETERS FOR CHANNEL MODELS CM1 AND CM2

\begin{tabular}{cc}
\hline Environment Residential & LOS (CM1.1) \& NLOS (CM2.1) \\
\hline Amplitude factor of LOS $\beta$ & 1 \\
Average number of clusters $L$ & 9 \\
Number of rays in cluster & random \\
Cluster arrival rate $\Lambda$ & $0.191 \mathrm{~ns}^{-1}$ \\
Ray arrival rate $\lambda$ & $1.22 \mathrm{~ns}^{-1}$ \\
Cluster decay factor $\Gamma$ & $4.46 \mathrm{~ns}$ \\
Ray decay factor $\gamma$ & $6.25 \mathrm{~ns}$ \\
LOS Component pathloss & $-82 \mathrm{~dB}$ \\
Distance between Tx and Rx & $5 \mathrm{~m}$ \\
Small Rician factor & 4.34 \\
Cluster lognormal standard deviation & $6.28 \mathrm{~dB}$ \\
Ray lognormal standard deviation & $13 \mathrm{~dB}$ \\
Angle spread & $49.8 \mathrm{degree}$ \\
\hline
\end{tabular}

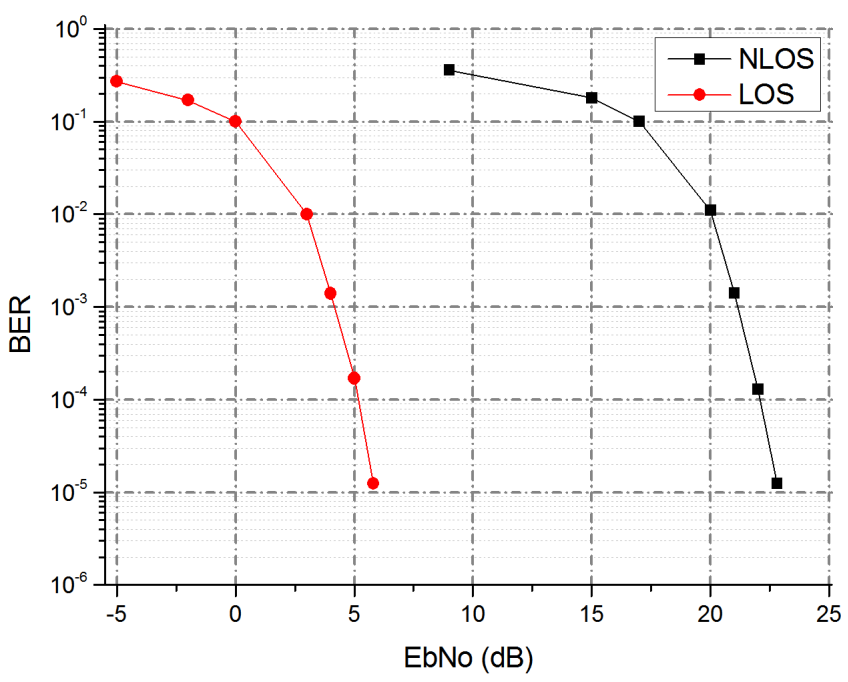

Fig. 13. Performance comparison between LOS and NLOS environment

CM1, We have a difference of $17 \mathrm{~dB}$ for a BER $=10^{-3}$. We can reduce this loss using beamforming antennas and MIMO technology.

\section{Analyzing the Effect of Modulation and Coding Schemes Order}

The HIS PHY mode consists of 12 different modulation and coding Schemes (MCS), with a maximum data rate equal to $5.8 \mathrm{Gbps}$, and support only LDPC codes. For our simulations we have considered three MCSs summarize in table III. By fixing MMF length to $300 \mathrm{~m}$ and varying $\mathrm{EbNo}$, we have considered the system in LOS environment with different modulation and coding schemes; MCS1, MCS4 and MCS7, as shown in Fig. 14. The performances of the system increase when the modulation orders decrease. The QPSK offers the best BER performances compared to 16QAM and 64 QAM. We observe a gain in the order of $7.5 \mathrm{~dB}$ for the MCS1 mode (QPSK) compared to the MCS7 mode (64QAM). For an EbNo greater than $10 \mathrm{~dB}$ we can reach a bit rate of the order of 5.8 $\mathrm{Gb} / \mathrm{s}$ with a Binary Error Rate less than $10^{-3}$. Due to the 
TABLE III

High SpeEd InTERFACE PHY MCSS PARAMETERS

\begin{tabular}{cccc}
\hline MCS index & Data rate $(\mathrm{Mb} / \mathrm{s})$ & Modulation scheme & LDPC codes rate \\
\hline 1 & 1540 & QPSK & $1 / 2$ \\
4 & 3080 & 16QAM & $1 / 2$ \\
7 & 5775 & 64QAM & $5 / 8$ \\
\hline
\end{tabular}

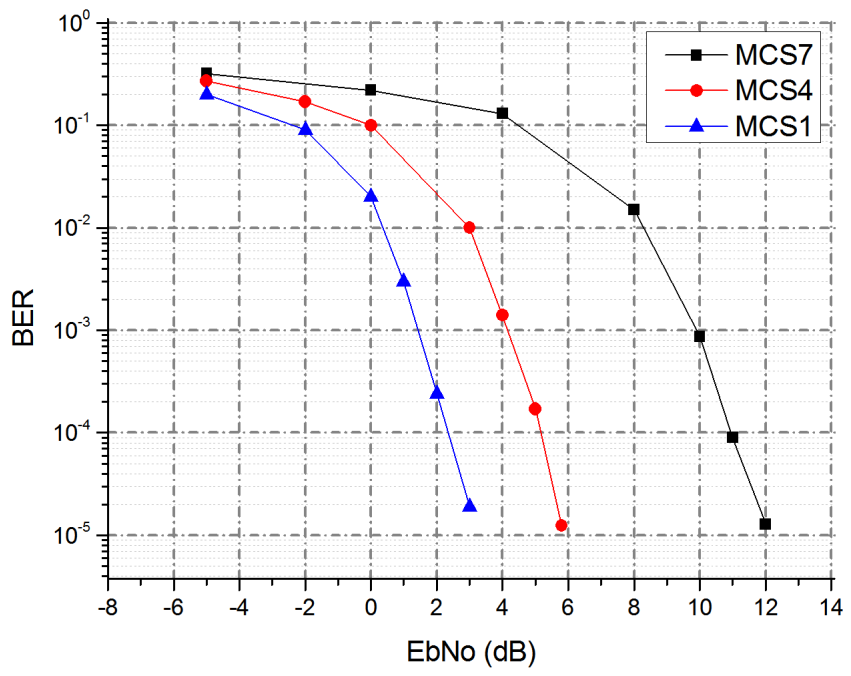

Fig. 14. BER plots of various modulation schemes in LOS environment

optical link transparency, our results are in good agreement with the results published in [23]

\section{CONCLUSION}

In this paper, we have presented and evaluated a complete WPAN HSI-OFDM RoF system at $60 \mathrm{GHz}$ using MMF and TG3c channel model in LOS and NLOS modes. We have shown that, by using the OCS modulation and LDPC codes, the MMF can be used to transport $60 \mathrm{GHz}$ OFDM over a distance up to $300 \mathrm{~m}$ followed by $5 \mathrm{~m}$ wireless transmission. Future work will consider adaptive modulation and coding schemes (MCS) based on the prediction of channel state information (CSI) in order to achieve a good compromise between high data rates and high transmission quality. And also, based on the TSV model, RoF-MIMO system could be considered to take advantage of the available bandwidth of the fiber.

\section{REFERENCES}

[1] "IEEE Standard for Information technology- Local and metropolitan area networks- Specific requirements- Part 15.3: Amendment 2: Millimeter-wave-based Alternative Physical Layer Extension", in IEEE Std 802.15.3c-2009 (Amendment to IEEE Std 802.15.3-2003), vol., no., pp.1-200, Oct. 122009.
[2] (2010, December) ECMA-387 High Rate $60 \mathrm{GHz}$ PHY MAC and HDMI PAL. [Online]. https://www.ecmainternational.org/publications/files/ECMA-ST/ECMA-387.pdf

[3] $(2010$, May) WirelessHD Specification Overview. [Online] http://www.wirelesshd.org/

[4] "IEEE Draft Standard for Local and Metropolitan Area Networks Specific Requirements - Part 11: Wireless LAN Medium Access Control (MAC) and Physical Layer (PHY) Specifications - Amendment 3: Enhancements for Very High Throughput in the $60 \mathrm{GHz}$ Band", in IEEE P802.11ad/D5.0, September 2011, (Draft Amendment based on IEEE P802.11REVmb D10.0) as amended by IEEE 802.11ae D5.0 and IEEE 802.11aa D6.0), vol., no., pp.1-601, Dec. 202011

[5] J. Guillory, E. Tanguy, et al., "A $60 \mathrm{GHz}$ Wireless Home Area Network With Radio Over Fiber Repeaters", Journal of Lightwave Technology, Vol. 29, pp. 2482-2488, 2011.

[6] C. H. Lee, Microwave Photonics, 2nd ed.: CRC Press, 2013.

[7] K. Yang, et al., "Transmission of $60 \mathrm{GHz}$ wired/wireless based on fullduplex radio-over-fiberusing dual-sextupling frequency”, IET Communications, Vol. 6, pp. 2900-2906, 2012.

[8] I. Gasulla, J.Capmany, "Phase-modulated radio over fiber multimode links", Optics Express, Vol. 20, pp. 11710-11717, 2012.

[9] I. B. Djordjevic, 'LDPC-Coded OFDM Transmission Over GradedIndex Plastic Optical Fiber Links", IEEE Photonics Technology Letters, Vol. 19, pp. 871-873, 2007.

[10] I. Dayoub, et al., "Radio-optic demonstrator for distributed antenna system indoor wireless applications using low-cost VCSELs', European Transactions on Telecommunications, Vol. 18, pp. 811-814, 2007.

[11] M. G. Larrode, et al., "Overcoming Modal Bandwidth Limitation in Radio-over-Multimode Fiber Links", IEEE Photonics Technology Letters, Vol. 18, pp. 2428-2430, 2006.

[12] X. Wei, et al., "Application of Turbo codes in optical OFDM multimode fiber communication system", Optics Communications, Vol. 281, pp. 1118-1122, 2008.

[13] M. Elyahyaoui, A. El Moussati, et al., "Performance evaluation of coherent optical OFDM communications using LDPC codes", International Journal of Microwave and Optical Technology, Vol. 11, pp. 72-79, 2016.

[14] G. Baghersalimi, et al., "Pilot-aided estimation and equalisation of a radio-over-fiber system in wideband code division multiple access", IET Communications, Vol. 7, pp. 999-1007, 2013.

[15] H. Sawada, et al., "Proposal of novel statistic channel model for millimeter wave WPAN", Asia-Pacific Microwave Conference, pp. 1855 1858, December 2006,

[16] S.K.Yong et al., "TG3c Channel Modeling Sub-committee Final Report," IEEE802.15-07-0584-01-003c, Mar. 2007.

[17] k. Charade, et al., "analysis of fi-wi indoornetwork architecture based on 802.15.3c", IEEE 11th Consumer Communications and Networking Conference Las Vegas, pp.101-106, January 2014.

[18] D. Pepe and D. Zito, Applications of Matlab in Science and Engineering: InTech Open Access, 2011.

[19] A. A. M. Saleh, Valenzuela, "A Statistical Model for Indoor Multipath Propagation", IEEE Journal on Selected Areas in Communications, Vol. 5, pp. 128-137, 1987.

[20] (2007) TG3C Channel model Matlab code. [Online] https://mentor.ieee.org/802.15/documents/

[21] J. Zhensheng, et al., "A full-duplex radio-over fiber system based on optical carrier suppression and reuse", IEEE Photonics Technology Letters, Vol. 18, pp. 17261728, 2006.

[22] R. E. Freund, et al.,"High-Speed Transmission in Multimode Fibers", Journal of Lightwave Technology, Vol. 28, pp. 569-586, 2010.

[23] M. Liso Nicolas, et al "Physical layer simulation results for IEEE 802.15.3c with differentchannel models", Advances in Radio Science, Vol. 9, pp.173-177, 2011 